\title{
Actualización: Abordaje quirúrgico de la obesidad
}

\author{
Surgical approach of obesity
}

Axel Beskow

\begin{abstract}
Resumen
En la obesidad mórbida, los tratamientos conservadores conocidos están condenados al fracaso en más del $97 \%$ de los casos. Su tratamiento quirúrgico persigue el objetivo de controlar la enfermedad a largo plazo reduciendo los riesgos y mejorando las comorbilidades. El candidato a cirugía bariátrica es un paciente de alto riesgo con "obesidad mórbida" de más de cinco años de evolución, con riesgo quirúrgico aceptable, estabilidad psiquiátrica y compromiso evidente con el programa de tratamiento. La evaluación prequirúrgica debe ser exhaustiva. El manejo de estos pacientes debe ser multidisciplinario, siendo los cambios conductuales los verdaderos responsables de un buen resultado alejado. En dicho contexto, la cirugía bariátrica posee baja morbimortalidad, la cual está determinada por la condición clínica del paciente. En este artículo, el autor describe sencillamente las técnicas "restrictivas" y "malabsortivas" de uso más frecuente, su efectividad y su riesgo de complicaciones.
\end{abstract}

\begin{abstract}
Morbid obesity known conservative treatments are doomed to fail in more than $97 \%$ of cases. Surgical treatment aims to control the disease long term by reducing its risks and improving its comorbidities. The bariatric surgery candidate is a high risk patient with "morbid obesity" over five years of evolution, with acceptable surgical risk, psychiatric stability and clear commitment to treatment program. Surgical candidates should be thoroughly evaluated. It requires multidisciplinary management, since behavioral changes are paramount for a good long term result. In this context, bariatric surgery has low morbidity and mortality, which are determined by the patient's clinical condition.

In this article, the author merely describes the "restrictive" and "malabsorptive" techniques most frequently used, their effectiveness and risk of complications.
\end{abstract}

Palabras clave: obesidad, cirugía baríatrica, efectividad. Key words: obesity, bariatric surgery, effectiveness.

Beskow A. Abordaje quirúrgico de la obesidad.Evid Act Pract Ambul. Vol 13(1).29-31. Ene-Mar 2010.

\section{Introducción}

La obesidad constituye un problema de salud cuya consulta es cada vez más frecuente. Algunos de los motivos que determinan este hecho son su prevalencia creciente, los problemas a los que se asocia -ej. diabetes tipo 2, limitación física e hipertensión arterial- los preconceptos socioculturales que estigmatizan a estos enfermos caracterizándolos como personas débiles y egoístas, la discriminación laboral, el temor a verse incapacitados y dependientes, la percepción negativa de su imagen corporal, etc. Afortunadamente, sólo una minoría de las personas obesas se encuentra en una situación clínica tan complicada como para requerir un abordaje quirúrgico. Son personas con un grado de obesidad importante -índice de masa corporal (IMC) mayor a $40 \mathrm{~kg} / \mathrm{m} 2$ - o con morbilidad generada por el exceso de tejido adiposo. Estos pacientes son "obesos mórbidos" y consultan, por lo general, luego de varios años de esfuerzos infructuosos para bajar de peso a través de planes alimentarios, ejercicio regular, fármacos, preparados de dudosa composición y otros tratamientos con un sustento aún más dudoso. Resulta lógico que estos pacientes consulten buscando bajar de peso aunque en realidad, en general, pretenden un verdadero cambio de vida. Para este grupo de pacientes la cirugía bariátrica, a pesar de sus riesgos, constituye una respuesta efectiva y segura.

\section{Epidemiología}

El problema de la obesidad a nivel mundial ha cobrado tal magnitud que se lo cataloga como una pandemia. Las estadísticas sobre obesidad y sobrepeso en diferentes países muestran que si bien la progresión inicial y más importante se dio en Occidente, los demás países, aunque unos pasos más atrás, han recorrido el mismo camino. La progresión es más marcada aún en la población infantil y adolescente. En este grupo las tasas de sobrepeso se han duplicado o triplicado en los últimos 15 años, y las de obesidad, cuadruplicado o quintuplicado, de forma tal que en 2004 cerca del 15\% de los niños y adolescentes presentaba sobrepeso y más del $5 \%$ obesidad (en algunos países superaba el $10 \%$ ). En la población adulta las tasas de sobrepeso son muy dispares, pero en países occiden- tales como Canadá o Estados Unidos se estima que cerca del $60 \%$ de la población presenta sobrepeso, y entre el $20 \%$ y el $25 \%$, obesidad. Si bien en Argentina las estadísticas son incompletas, en una encuesta nacional del año 2003 sobre 1289 jóvenes de 10 a 19 años se determinó una prevalencia de sobrepeso del $20,8 \%$ y una de obesidad del $5,4 \%$. En un relevamiento publicado por la Sociedad Argentina de Cardiología en 2002 sobre 14.584 mayores de 18 años, el 36\% presentaba sobrepeso y el $17 \%$ obesidad. Por otro lado, la Encuesta Nacional de Nutrición y Salud 2004-2005 realizó más de 36.000 encuestas en niños y en mujeres en edad fértil: el 6,6\% de los niños de seis meses a cinco años presentaba obesidad (8,3\% en el Gran Buenos Aires) mientras que el $24,9 \%$ de las mujeres presentaba sobrepeso y el $19,4 \%$, obesidad. En los grandes centros urbanos el problema es aún mayor y se estima que hoy en día en Argentina, más del $55 \%$ de la población tiene sobrepeso $u$ obesidad, mientras que entre el 3 y el $5 \%$ de la población es obesa mórbida.

\section{Fisiopatogenia}

La obesidad es probablemente una de las enfermedades más desconocidas y complejas. Los cambios en el estilo de vida del hombre en las últimas décadas -sedentarismo, televisión, informatización, disponibilidad de alimentos y calidad de los mismos- parecieran ser los principales responsables de la epidemia. Si bien no hay dudas sobre la predisposición genética a la obesidad en un buen porcentaje de casos, los factores adquiridos generan una presión particular sobre nuestra genética que ha favorecido la acumulación calórica en depósitos tisulares. La predisposición de nuestro metabolismo es a mantener nuestro peso y, de ser posible, a aumentarlo.

Evidentemente son múltiples los caminos que conducen al incremento del apetito cuando se realizan dietas hipocalóricas, ya que ningún fármaco ha logrado modificar significativamente esta respuesta adaptativa. A pesar de esto, cuando existe sobrepeso u obesidad leve, los tratamientos conservadores tienen buenas expectativas de resultado. Sin embargo, en la obesidad mórbida, los tratamientos conservadores conocidos están condenados al fracaso en más del $97 \%$ de los casos. 


\section{Presentación clínica}

No todo enfermo obeso es candidato a cirugía. Únicamente lo es aquel paciente con "obesidad mórbida" de más de cinco años de evolución, con riesgo aceptable, estabilidad psiquiátrica y compromiso evidente con el programa de cirugía bariátrica. El consenso del Instituto Nacional de Salud de EEUU de 1991 estableció los criterios de inclusión, que se enumeran en el cuadro 1. Por ejemplo, ningún paciente con adicciones activas, enfermedad oncológica no controlada, enfermedad metabólica no compensada, coagulopatía, hipertensión portal o inestabilidad psiquiátrica debería operarse.

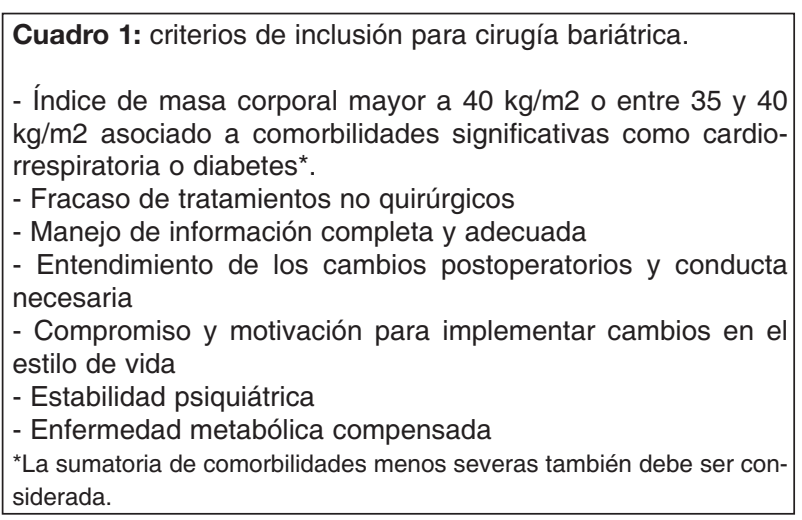

El candidato a cirugía es en general un paciente de alto riesgo, no sólo por la obesidad sino por las severas comorbilidades manifiestas al momento de la consulta. En las series quirúrgicas, cerca del $30 \%$ de los enfermos son diabéticos, $50 \%$ padecen apnea de sueño y más del $50 \%$ son hipertensos. La obesidad central o androide es la que predispone en mayor medida al desarrollo de comorbilidades severas, particularmente las metabólicas y cardiorrespiratorias. Las comorbilidades más frecuentes se enumeran en el cuadro 2.

Cuadro 2: principales enfermedades asociadas a la obesidad.

- Dislipemias: hipercolesterolemia, hipertrigliceridemia, aumento del colesterol LDL y disminución del HDL

- Diabetes mellitus tipo 2 e insulinorresistencia.

- Hipertensión arterial.

- Cardiopatía isquémica, insuficiencia cardíaca y accidente cerebrovascular.

- Alteraciones osteoarticulares: artritis deformante (miembros inferiores).

- Insuficiencia venosa en miembros inferiores.

- Hiperuricemia y gota.

- Enfermedades digestivas: esteatosis hepática, reflujo gastroesofágico y litiasis biliar

- Apnea/hipopnea del sueño.

- Insuficiencia respiratoria.

- Trastornos psicológicos: depresión.

- Alteraciones cutáneas.

- Tumores malignos: colon, recto, próstata, ovarios, endometrio y mama.

Cabe preguntarse si no debiera indicarse la cirugía antes del desarrollo de las comorbilidades severas, aún en casos con IMC menores de $35 \mathrm{~kg} / \mathrm{m} 2$, siempre y cuando se hayan agotado los tratamientos conservadores, hecho que seguramente reduciría el riesgo quirúrgico. No existe consenso sobre este tema y cada caso debe ser analizado individualmente sopesando riesgos y beneficios.

\section{Sistemática de estudio}

La evaluación global se llevará a cabo por parte de un equipo multidisciplinario cuyos pilares son el cirujano, el médico nutricionista y el psiquiatra. Los pacientes que son considerados para cirugía bariátrica requieren estudios generales para documentar sus comorbilidades, y para evaluar su riesgo quirúrgico, su estado de salud general y su estado de salud digestivo. Lo primero se logra a través de un exhaustivo interrogatorio sobre la historia de su obesidad, las comorbilidades, los hábitos alimentarios, los antecedentes psiquiátricos y los antecedentes familiares. Se toman diferentes medidas antropométricas (peso, talla, IMC, circunferencia de la cintura, circunferencia de la cadera, pliegue cutáneo) y se realiza una impedancia bioeléctrica. Se registra la tensión arterial y se evalúan lesiones cutáneas y subcutáneas, así como el abdomen. Por ejemplo, muchos pacientes han tenido cirugías abdominales que pueden dificultar el abordaje laparoscópico.

Se solicita una endoscopía digestiva alta con el fin de evaluar el esófago, el estómago y tomar una biopsia para determinación de Helicobacter Pylori. Son críticas las evaluaciones cardiológica, respiratoria y anestésica, y en la mayoría de los pacientes deber debe realizarse además un ecocardiograma doppler. La prueba de esfuerzo no siempre puede realizarse ya que muchos pacientes superan el peso máximo tolerado por los equipos. La polisomnografía sólo es mandatoria ante clínica sospechosa de apnea de sueño severa (somnolencia diurna) y que podría requerir el uso de CPAP. Se solicitará un laboratorio completo incluyendo insulinemia basal, hemoglobina glicosilada, tirotrofina (TSH) y perfil de coagulación.

En todos los casos debe realizarse una evaluación psiquiátrica, idealmente por un profesional integrante del equipo multidisciplinario. La conclusión de la evaluación deberá determinar la pertinencia y la oportunidad de la cirugía.

\section{Tratamiento}

El tratamiento quirúrgico de la obesidad mórbida persigue el objetivo de controlar la enfermedad a largo plazo reduciendo los riesgos y mejorando las comorbilidades. Este ambicioso objetivo se logra ayudando a controlar el apetito, generando saciedad precoz y estimulando al paciente para incorporar conductas de alimentación saludable y ejercicio regular. Una cirugía por sí misma no lograría cumplir con estos mecanismos, por lo cual se requiere del manejo multidisciplinario a largo plazo, con una verdadera terapia cognitivo-conductual.

El proceso quirúrgico implica tres etapas: 1) la preparación del paciente -predisposición, información y dieta preoperatoria inmediata; 2) la cirugía misma, con los cuidados anestésicos y perioperatorios que requieren de un marco institucional de alta complejidad; 3) el seguimiento postoperatorio quirúrgico y nutricional a largo plazo. Resultan de gran ayuda los grupos de apoyo formados por pacientes y profesionales.

\section{Técnicas quirúrgicas}

Existen diferentes técnicas quirúrgicas que se dividen en restrictivas y malabsortivas, según su principal mecanismo de acción. Entre las restrictivas se destacan la banda gástrica ajustable (BGA) que se describe en la figura 1, el bypass gástrico en $Y$ de Roux (BGYR) visible en la figura 2; y la manga gástrica (MG) que puede observarse en la figura 3. 
Figura 1: esquema de banda gástrica ajustable.

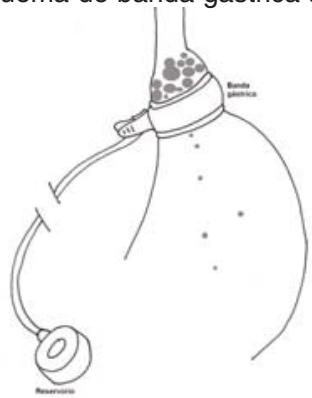

Figura 2: esquema de Bypass gástrico en "Y de Roux".

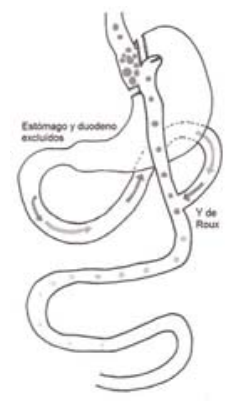

Figura 3: esquema de gastrectomía "en manga".

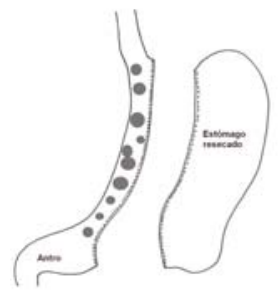

En todas ellas, se busca lograr la saciedad precoz, generando un neoestómago de 15 a $20 \mathrm{~cm} 3$. En las dos últimas se agrega un efecto anorexígeno que se explica por modificaciones endocrinas digestivas (grelina, GLP-1, PYY, etc.). El BGYR posee un componente malabsortivo relativo, salvo que se trate de un "bypass de asa larga" en cuyo caso más de dos metros de yeyuno dejan de tener función absortiva. Los procedimientos malabsortivos más comunes son la derivación biliopancreática (DBP) y el switch duodenal (SD). En éstos se reduce parcialmente la capacidad gástrica y se construyen un asa alimentaria y un asa biliopancreática muy largas, con un asa común de 50 a $75 \mathrm{~cm}$. El corto segmento con capacidad absortiva genera una verdadera malabsorción, hecho que restringe este tipo de procedimientos a enfermos con un control muy cercano. En términos generales, las cirugías restricti- vas obtienen un porcentaje de exceso de peso perdido (\%EPP) promedio del $50 \%$ al $80 \%$, dependiendo del tipo de cirugía y del grado de obesidad preoperatorio. Las cirugías malabsortivas obtienen un 80 a $90 \%$ de $\%$ EPP promedio, mientras que las comorbilidades mejoran en altos porcentajes. La BGA posee muy pocas complicaciones inmediatas pero a largo plazo cerca del $30 \%$ de los pacientes tendrán algún problema, incluyendo un descenso de peso insatisfactorio. Las cirugías con derivaciones intestinales (BGYR, DBP y SD) generan déficit absortivo de micronutrientes y obligan a la suplementación de vitaminas y minerales de por vida. En la DBP y el SD son frecuentes la esteatorrea y los déficits nutricionales. Las complicaciones generales (trombosis venosa profunda y tromboembolismo pulmonar, pancreatitis, infarto agudo de miocardio, insuficiencia respiratoria, etc.) son poco frecuentes pero conllevan riesgo de vida. La mortalidad de la cirugía bariátrica laparoscópica oscila entre el $0,2 \%$ (BGA) y el 2 al $3 \%$. Recientemente se ha destacado la efectividad de la cirugía como el BGYR en la resolución del la diabetes mellitus tipo 2 , lo que generó el concepto de "cirugía metabólica". Si bien este concepto es promisorio, cabe recordar que los riesgos quirúrgicos son significativos ya que la morbilidad de las cirugías con resecciones y anastomosis es infrecuente pero potencialmente grave. Por ejemplo, las fístulas digestivas se presentan en el 1 al $5 \%$ de los casos, siendo rara la hemorragia postoperatoria.

\section{Consejo y educación}

El abordaje quirúrgico de la obesidad está indicado en aquellas condiciones en que la obesidad constituye un serio problema de salud y un riesgo de muerte precoz. La cirugía posee efectos muy importantes sobre la saciedad y el apetito. Sin embargo, el objetivo buscado por este tipo de modalidad terapéutica es un verdadero cambio en el estilo de vida del enfermo, quien deberá comprometerse en su plan alimentario y en la incorporación de una actividad física regular. El seguimiento a largo plazo es fundamental. No todas las cirugías generan los mismos efectos y es por ello que el especialista, en base a las características del paciente, deberá elegir el mejor procedimiento para cada caso. En internet existe mucha información, no toda válida y la mayoría manejada por intereses particulares. Un sitio con información útil es http://www.asmbs.org, aunque lamentablemente se encuentra en inglés y no posee versión en español. También existe información básica en http://es.wikipedia.org/wiki/Cirugía_bariátrica.

\section{Interconsulta}

El paciente que es considerado para cirugía bariátrica debe ser derivado a un equipo multidisciplinario especializado. Si bien el seguimiento clínico a largo plazo puede ser llevado a cabo por el médico de cabecera, es aconsejable una visita al menos anual con el equipo.

Recibido el 07/12/09 y aceptado el 25/01/10

\section{Bibliografía recomendada}

Adams T y col. Long-Term Mortality after Gastric Bypass Surgery. N Engl J Med 2007; 357(8):753-61.

Balsiger B y col. Prospective Evaluation and 7-Year Follow-up of Swedish Adjustable Gastric Banding in Adults with Extreme Obesity. J Gastrointest Surg 2007; 11(11):1470-1477.

Balsiger B y col. Prospective Evaluation and 7-Year Follow-up of Swedish Adjustable Gastric Banding in Adults with Extreme Obesity.

Bazian Ltd. Surgery for morbid obesity: which technique works best? Evidence-Based Healthcare \& Public Health 2005; 9(4):284-291.

DeMaria E. Bariatric Surgery for Morbid Obesity. N Engl J Med 2007; 356(21):2176-83.

Diniz M y col. Gut-brain communication: how does it stand after bariatric surgery? Curr Opin Clin Nutr Metab Care 2006; 9:629-636.

Elder K y col. Bariatric Surgery: A Review of Procedures and Outcomes. Gastroenterology 2007; 132(6): 2253-2271.

Hainer V y col. Treatment Modalities of Obesity. What fits whom? Diabetes Care 2008; 31(Suppl 2): S269-S277.

Higa K y col. Complications of the Laparoscopic Roux-en-Y Gastric Bypass: 1,040 Patients - What Have We Learned? Obes Surg 2000; 10(6): 509-513.

Himpens J y col. A Prospective Randomized Study Between Laparoscopic Gastric Banding and Laparoscopic Isolated Sleeve Gastrectomy: Results after 1 and 3 Years. Obes Surg 2006; 16(11): 1450-1456. $\mathrm{NIH}$ conference. Gastrointestinal surgery for severe obesity. Consensus Development Conference Panel. Ann Intern Med 1991;115(12):956-61.

Ogden C y col. The Epidemiology of Obesity. Gastroenterology 2007;132(6):2087-2102.

Parikh M y col. Hajiseyedjavadi O, Ren CJ. Objective Comparison of Complications Resulting from Laparoscopic Bariatric Procedures. J Am Coll Surg 2006; 202(2): 252-261.

Pories W. Bariatric Surgery: Risks and Rewards. J Clin Endocrinol Metab 2008; 93(11): S89-S96.

Sjöström L y col. Effects of Bariatric Surgery on Mortality in Swedish Obese Subjects. N Engl J Med 2007; 357(8):741-52.

Weiner R y col. Outcome after Laparoscopic Adjustable Gastric Banding - 8 Years Experience. Obes Surg 2003; 13(3), 427-434.

Wren A y col. Gut Hormones and Appetite Control. Gastroenterology 2007;132(6):2116-2130. 